\title{
Exercício Físico e Qualidade de Vida: um Estudo de Delineamento Quase-Experimental com Estudantes e Servidores Universitários
}

\author{
Physical Exercise and Quality of Life: A Quasi-Experimental Study with College \\ Students and University Staff
}

\author{
Everton Gonçalves Macedo ${ }^{1}$ \\ Yuri da Silva Teixeira ${ }^{1}$ \\ Augusto Doninni Akkari ${ }^{1}$ \\ Janaina Nunes de Oliveira ${ }^{2}$ \\ Charles Jhonatan Martins de Andrade ${ }^{2}$ \\ Daniel Delani ${ }^{3}$ \\ Silvia Teixeira de Pinho ${ }^{4}$ \\ Tatiane Gomes Teixeira ${ }^{4}$
}

\section{RESUMO}

Objetivo: Investigar os efeitos de oito semanas de caminhada sobre a qualidade vida (QV) da comunidade universitária. Metodologia: Pesquisa quase-experimental, com participação de 17 estudantes e quatro servidores da Universidade Federal de Rondônia. Os participantes foram organizados em grupos, conforme assiduidade (ASD) no programa de caminhada: G40M (ASD>40\%; $=9$ ); e GA35 (ASD<35\%; $n=6$ ) e SE (sem exercício; $n=6$ ). As sessões de caminhada foram realizadas três vezes por semana, durante oito semanas, com intensidade progressivamente aumentada ao longo das sessões. A QV foi mensurada pelo questionário SF-36, que avalia esta variável em oito domínios: capacidade funcional, aspectos físicos, aspectos emocionais, aspectos sociais, dor, vitalidade, estado geral de saúde e saúde mental. Foram utilizadas ferramentas de estatística descritiva e inferencial para testar diferenças entre os grupos ou momentos, adotando-se $(p<0,05)$ para definição de significância estatística. $O$ tamanho do efeito (TE) também foi calculado. Resultados: No G40M o domínio aspectos emocionais foi o que sofreu mudança numérica mais expressiva (pré: $55,53 \pm 40,82$; pós: $77,75 \pm 28,88$ ), sem diferença estatística, com TE moderado. Para os demais domínios também não foram encontradas diferenças estatísticas (pré versus pós). No GA35 seis domínios sofreram redução, mas sem diferença estatística. Os maiores valores de TE da pesquisa foram encontrados no SE $(1,14$ : estado geral de saúde; -1,05: vitalidade), com elevação dos escores médios em ambos. Conclusão: O programa de caminhada não demonstrou impacto expressivo na QV dos voluntários, mas a pesquisa evidenciou a importância de estudos experimentais/quaseexperimentais para investigar a relação entre a QV e a prática de exercícios, especialmente na comunidade universitária.

\section{DESCRITORES}

Qualidade de vida. Saúde. Exercício físico. Caminhada. Universidades.

\begin{abstract}
Objective: The research aimed to investigate the effects of eight weeks of walking on the quality of life (QOL) of servants and college students. Methods: Quasi-experimental study, with 17 students and 4 staff members of the Federal University of Rondônia. Participants were organized into groups, according to attendance (ASD) in the exercise sessions: G40M (ASD of $40 \%$ or more; $n=9$ ); GA35 (ASD below $35 \% ; n=6$ ) and SE (without exercise; $n=6$ ). The walk was carried out three times a week, for eight weeks, with progressively increased intensity. QOL was evaluated by SF-36. This questionnaire assesses this variable in eight domains: functional capacity, physical aspects, pain, general health, vitality, social functioning, emotional aspects and mental health. Descriptive and inferential statistics were used to test differences between groups or moments, and $p$ values $<0,05$ were considered statistically significant. Effect size (TE) was calculated too. Results: In the G40M, the emotional aspects domain had the most significant numerical changes (pre: $55,53 \pm 40,82$; post: $77,75 \pm 28,88$ ), without statistical difference and moderate TE. For the other domains, there were also no statistical differences (pre vs post). In GA35, six domains were reduced, but without statistical differences. The largest TE was found in SE (1,14: general health; -1,05: vitality), with an increase in the mean scores in both. Conclusion: The walk program did not demonstrate a significant impact on the QOL of the volunteers, but the research showed the importance of experimental/quasi-experimental studies to investigate the relationship between QOL and physical exercise, especially in the university community.
\end{abstract}

DESCRIPTORS

Quality of Life. Health. Physical Exercise. Walking. Universities.

${ }^{1}$ Graduado pela Universidade Federal de Rondônia, Departamento de Educação Física, Porto Velho, Rondônia, Brasil

2 Estudante da Universidade Federal de Rondônia, Departamento de Educação Física, Porto Velho, Rondônia, Brasil.

${ }^{3}$ Professor da Universidade Federal de Rondônia, Departamento de Educação Física, Porto Velho, Rondônia, Brasil.

${ }^{4}$ Professora da Universidade Federal de Rondônia, Departamento de Educação Física, Porto Velho, Rondônia, Brasil. 
$P^{\prime}$ raticar atividades físicas regularmente é um hábito reconhecidamente benéfico à saúde ${ }^{1}$, gerador de impacto positivo em inúmeros sistemas orgânicos, bem como na prevenção e no tratamento de doenças ${ }^{2,3}$. Benefícios desta prática também são observados na saúde mental, no desempenho cognitivo $^{1}$ e em medidas subjetivas de satisfação com a vida, o bem-estar ${ }^{4}$ e a qualidade de vida ${ }^{5,6}$. Neste cenário, está bem estabelecido na literatura ${ }^{7}$ que se exercitar com regularidade, ou até mesmo ampliar a movimentação corporal no dia a dia, repercute de forma positiva em inúmeros aspectos da vida humana, especialmente, entre os sujeitos envolvidos, predominantemente, em atividades sedentárias no seu cotidiano.

Estudantes e servidores universitários compõem um grupo que merece atenção em relação à inatividade física, visto que, além permanecerem por longos períodos em atividades sedentárias ${ }^{8,9}$, a presença de ansiedade, do estresse e de outros hábitos não saudáveis ${ }^{10,11}$ são frequentes entre esses. Ademais, pesquisas revelam que o ambiente universitário não estimula a prática de atividades físicas ${ }^{12,13}$. Considerando os conhecidos efeitos de tal prática é importante que medidas favorecedoras de um estilo de vida ativo entre universitários sejam estimuladas, inclusive porque tal estratégia tem o potencial de colaborar para que os jovens estudantes adquiram e mantenham hábitos saudáveis ao longo da vida adulta.

Neste contexto, uma variável que merece atenção, especialmente em decorrência da relação que apresenta com a saúde ${ }^{12} \mathrm{e}$ do fato de ser potencialmente impactada pela prática de atividades físicas $^{8,14}$ é a qualidade de vida (QV). Ao longo das últimas três décadas o construto de QV foi amplamente discutido na literatura, consolidando-se o entendimento de sua característica multidimensional e subjetiva ${ }^{15,16}$. Em outras palavras, não existe uma definição única para $\mathrm{QV}^{17}$, porém, existe consenso que ela é afetada pelas múltiplas dimensões da vida humana e que sofre influência de aspectos individuais e culturais.

O conceito adotado pela Organização Mundial da Saúde, amplamente citado na literatura estabelece que a QV se refere à percepção do indivíduo sobre sua posição na vida, no contexto da cultura e do sistema de valores nos quais ele vive e em relação aos seus objetivos, expectativas, preocupações e padrões ${ }^{18}$. Minayo ${ }^{19}$ apresenta a QV como uma noção correspondente ao grau de satisfação encontrado na vida familiar, amorosa, social e ambiental e à própria estética existencial e que pressupõe a capacidade de efetuar uma síntese cultural de todos os elementos que determinada sociedade considera seu padrão de conforto e bem-estar. Dada a complexidade e diversidade conceitual da $Q V$, é fundamental que os pesquisadores apresentem nas publicações seu entendimento sobre tal construto ${ }^{17}$, viabilizando discussões mais pertinentes e contextualizadas.

Na presente pesquisa a investigação foi designada para compreender, a partir da perspectiva de estudantes e servidores universitários, se um programa de caminhada tem impacto sobre dimensões físicas, emocionais e sociais da vida de tal grupo. Assim, foi explorada a qualidade de vida relacionada à saúde (QVRS), a qual é considerada, no contexto de avaliação 
da QV, como o principal indicador para avaliar o resultado de intervenções ${ }^{19}$. Para tanto optou-se por utilizar um instrumento classificado como genérico para a medida da QVRS global: o questionário Medical Outcomes Survey - Short Form- 36 (SF-36) ${ }^{20}$. Em resumo, a variável principal do estudo é aqui entendida pelos pesquisadores como a medida da percepção dos sujeitos sobre sua saúde e seu bem-estar ${ }^{14.16}$.

Estudos prévios evidenciam que universitários fisicamente ativos apresentam escores mais elevados de QV, quando comparados aos sedentários ${ }^{21,22}$, sugerindo que a prática regular de exercícios tem efeitos positivos sobre essa variável. Embora seja significativo o número de publicações sobre tal temática, poucas realizaram intervenção com exercícios ${ }^{6}$. No Brasil, somente uma publicação apresenta resultados de um programa de intervenção com exercícios realizados com sujeitos da comunidade universitária ${ }^{23}$. Esta, porém, não incluiu estudantes. Assim, é relevante investigar e compreender se a realização de exercícios físicos afeta de forma direta a QV deste público. O presente estudo faz parte deste cenário e teve como objetivo principal identificar se a prática de caminhada durante oito semanas afetaria os escores de QV de estudantes e servidores universitários.

\section{METODOLOGIA}

Esta pesquisa teve abordagem quantitativa e caracterizou-se como quaseexperimental. A variável independente foi o programa de caminhada e a variável dependente foi a QV. O estudo atendeu as exigências das diretrizes e normas da pesquisa envolvendo seres humanos e foi aprovado no Comitê de Ética em Pesquisa da Universidade Federal de Rondônia (UNIR), sob o Parecer de $n^{\circ}$. 1.954.535.

A amostra foi não-probabilística e definida por conveniência. O recrutamento dos voluntários se deu por meio de divulgação no Campus universitário e nos endereços digitais a ele vinculados. Foi realizado de janeiro a março de 2017, na UNIR, em Porto Velho. Foram critérios para inclusão na pesquisa: idade entre 19 e 59 anos e ser pertencente ao quadro discente ou de servidores da UNIR. Os critérios de exclusão foram: a) presença de doenças cardiovasculares, metabólicas ou ostemioarticulares que pudessem interferir nos resultados da pesquisa ou cuja condição clínica exigisse cuidados especiais para a realização de exercícios físicos, tais como: hipertensão arterial sistêmica, diabetes, doenças pulmonares, osteoartrite, osteoporose entre outras; b) inaptidão para prática de exercícios, identificada em exame cardiológico; c) realização regular de atividades físicas nos três meses anteriores ao início do estudo; d) não concordância com alguma das etapas da pesquisa; e/ou e) não assinatura do termo de consentimento livre e esclarecido.

A amostra foi composta por dezessete estudantes e quatro servidores da UNIR, totalizando vinte e um (21) voluntários. Desse total, dezessete eram mulheres. A idade média dos voluntários foi de 27,24 $\pm 8,19$ anos e o IMC 25,18 $\pm 4,60 \mathrm{~kg} / \mathrm{m}^{2}$. As análises pré-exercício foram realizadas em abril; o programa de exercícios de abril a junho e as análises finais (após 8 semanas), em junho de 2017. Devido as grandes diferenças de 
assiduidade entre os participantes do grupo experimental, após o programa de exercícios, este grupo foi reorganizado segundo assiduidade no programa de caminhada: grupo com assiduidade igual ou inferior a 35\% (GA35) e grupo com assiduidade de $40 \%$ ou mais (G40M).

O programa de treinamento de caminhada foi realizado no Campus de Porto Velho da UNIR e teve duração de 8 semanas, com frequência semanal de três vezes. As sessões tiveram duração de 30 a 60 minutos e incluíram três momentos: aquecimento $(5 \mathrm{a}$ 10'), caminhada (20 a 40') e a volta a calma (5 a 10'). Tanto o aquecimento quanto a volta a calma consistiram em atividades dinâmicas de baixa intensidade, envolvendo grandes grupos musculares. $O$ primeiro teve objetivo de preparar o organismo para a prática de caminhada, enquanto, à volta a calma teve como objetivo promover o retorno das variáveis fisiológicas aos valores próximos ao repouso.

A caminhada foi realizada dentro dos limites de intensidade recomendados pelo American College of Sports Medicine $(\mathrm{ACMS})^{24}$ : moderada a vigorosa. A intensidade moderada compreende $40 \%$ a $59 \%$ da frequência cardíaca de reserva (FCreserva) e/ou valores de 12 a 13 na escala de Borg. A intensidade vigorosa compreende $60 \%$ a $84 \%$ da frequência cardíaca de reserva e valores de 14 a 16 na escala de Borg. As frequências cardíacas de treinamento e de reserva, respectivamente, foram calculadas por meio das seguintes equações: [FCtreinamento $=$ FCrepouso + (FCreserva $x$ Intensidade de treinamento)]; [FCreserva $=$ FCmáxima FCrepouso].
A FCrepouso foi considerada a menor frequência cardíaca mensurada com o avaliado em posição sentada, após este ter permanecido nesta posição durante 10 minutos, em ambiente calmo e silencioso. Durante as sessões de treinamento as intensidades foram controladas mediante medida da FC pelo próprio indivíduo por palpação da artéria radial e informada ao instrutor responsável. Ademais, foi utilizada a tabela adaptada de classificação de percepção subjetiva de esforço de Borg. Durante a realização da caminhada os sujeitos deveriam estar classificados na intensidade moderada ou vigorosa, dependendo do estágio de treinamento, em ambos os critérios. A progressão do treinamento foi realizada de acordo com as recomendações do ACSM ${ }^{24}$.

A QV foi avaliada pelo Short Form Health Survey-36 (SF-36), questionário composto por 36 itens analisados em oito domínios: capacidade funcional, aspectos físicos, dor corporal, estado geral de saúde, vitalidade, aspectos sociais, aspectos emocionais e saúde mental. A pontuação varia de 0 a 100 em cada domínio. Quanto maior a pontuação, melhor a condição de saúde relacionada à $\mathrm{QV}^{25}$.

Os dados foram analisados quantitativamente a partir da estatística descritiva e inferencial, utilizando o software ActionStat $₫$. Para comparações dos valores entre os momentos pré e pós-treinamento foi utilizado o teste de Wilcoxon pareado. Para comparar os grupos foi utilizado o teste de Kruskal Wallis e pós-hoc de Bonferroni, utilizando os valores dos deltas. Estes foram calculados pela subtração dos valores póstreinamento pelos valores pré-treinamento. Foi 
adotado como valor de significância estatística $p \leq 0,05$. Também foi calculado o tamanho do efeito (TE), comparando momentos pré versus pós intragrupos, utilizando o cálculo d de Cohen. Foram considerados efeitos insignificantes valores inferiores a 0,19 ; pequenos os valores de 0,20 a 0,49; médios valores entre 0,50 e 0,79; grandes de 0,80 a 1,29 ; e muito grandes valores acima de $1,30^{26}$.

\section{RESULTADOS}

Na Tabela 1 constam as características dos participantes, segundo os grupos. A maioria dos sujeitos era formada por estudantes, do sexo feminino e pertencente à faixa etária inferior a 30 anos.

$\mathrm{Na}$ Tabela 2 constam as médias dos valores pré e pós 8 semanas para cada um dos grupos. Diferenças significativas no momento pré-intervenção foram encontradas para as variáveis: dor (SE menor que G40M) e estado geral de saúde (SE menor que GA35). No momento pós-intervenção tais diferenças desapareceram, o que ocorreu fundamentalmente por elevação dos escores/ médias no grupo SE, sugerindo que tais domínios da QV estavam baixos no momento pré e se normalizaram, ou seja, alcançaram os valores encontrados no GA35 e G40M, no momento pós.

Quando os grupos foram comparados somente no momento pós-intervenção, foram encontradas diferenças nos domínios: capacidade funcional ( $G 40 \mathrm{M}$ maior que demais grupos), vitalidade (GA35 menor que SE) e saúde mental (GA35 menor que SE e G40M).

Nas análises intragrupos (pré versus pós-intervenção) nenhuma diferença significativa foi encontrada no G40M; e o tamanho do efeito variou entre pequeno (dor, estado geral de saúde, saúde mental) e médio (capacidade funciona, aspectos físicos, vitalidade, aspectos sociais, aspectos emocionais) no referido grupo.

Ainda sobre as análises de TE, merece destaque o domínio aspectos emocionais, enquanto, no G40M o efeito foi médio $(-0,63)$, nos grupos SE e GA35 foi insignificante (ambos <0,19).

$\mathrm{Na}$ Tabela 3 constam os deltas

Tabela 1. Número e percentual de sujeitos por sexos, faixa etária e categoria segundo grupos

\begin{tabular}{|c|c|c|c|c|}
\hline \multirow{3}{*}{\multicolumn{2}{|c|}{ Variáveis }} & SE & $\begin{array}{l}\text { GA35 } \\
n=21\end{array}$ & G40M \\
\hline & & $(n=6)$ & $(n=6)$ & $(n=9)$ \\
\hline & & \multicolumn{3}{|c|}{$\mathrm{n}(\%)$} \\
\hline Sexo & Feminino & $05(83,3)$ & $05(83,3)$ & $7(77,7)$ \\
\hline & Masculino & $01(16,67)$ & $01(16,67)$ & $2(22,2)$ \\
\hline Categoria & Estudantes & $04(66,6)$ & $05(83)$, & $8(88,8)$ \\
\hline & Servidores & $02(33,3)$ & $01(16,67)$ & $1(11,1)$ \\
\hline Faixa etária & $\begin{array}{l}20-29 \\
30-39 \\
40-45\end{array}$ & $\begin{array}{c}05(83,3) \\
01(16,67) \\
-\end{array}$ & $\begin{array}{c}03(50) \\
02(33,3) \\
01(16,67)\end{array}$ & $\begin{array}{l}6(66,6) \\
2(22,2) \\
1(11,1)\end{array}$ \\
\hline
\end{tabular}


Tabela 2. Média e desvio-padrão dos domínios de qualidade de vida dos estudantes e servidores universitários nos momentos pré e pós-intervenção

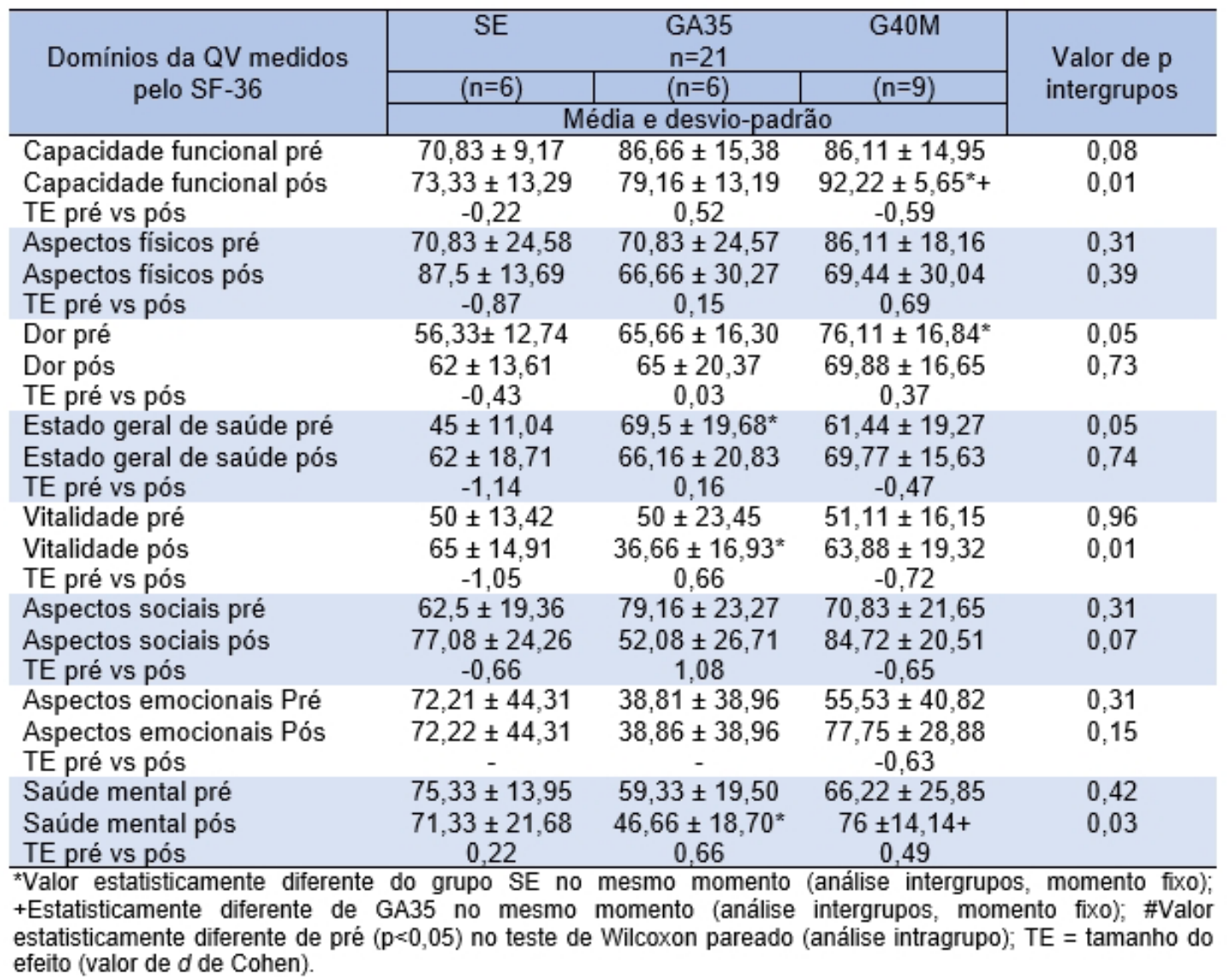

Tabela 3. Média e desvio-padrão dos domínios de QV de estudantes e servidores universitários nos momentos pré e pós

\begin{tabular}{|c|c|c|c|c|}
\hline \multirow{3}{*}{ Variáveis } & SE & $\begin{array}{l}\text { GA35 } \\
n=21\end{array}$ & G40M & \multirow{3}{*}{$\begin{array}{c}\text { Valor de } p \\
\text { intergrupost }\end{array}$} \\
\hline & $(n=6)$ & $(n=6)$ & $(n=9)$ & \\
\hline & \multicolumn{3}{|c|}{ Média e desvio-padrão } & \\
\hline Capacidade funcional & $2,5 \pm 18,91$ & $-7,5 \pm 14,05$ & $6,11 \pm 17,81$ & 0,26 \\
\hline Aspectos físicos & $16,67 \pm 20,41$ & $-4,16 \pm 10,20$ & $-16,66 \pm 37,5$ & 0,10 \\
\hline Dor & $5,7 \pm 18$ & $-0,66 \pm 21,57$ & $-6,22 \pm 24,72$ & 0,82 \\
\hline Estado geral de saúde & $17 \pm 12,96$ & $-3,33 \pm 6,83^{*}$ & $8,33 \pm 12,74$ & 0,02 \\
\hline Vitalidade & $15 \pm 21$ & $13,33 \pm 22,28$ & $12,77 \pm 24,76$ & 0,08 \\
\hline Aspectos sociais & $14,58 \pm 26,71$ & $-27,08 \pm 35,72$ * & $13,88 \pm 17,05$ & 0,04 \\
\hline Aspectos emocionais & $0 \pm 76,02$ & $0,05 \pm 36,47$ & $22,22 \pm 40,83$ & 0,72 \\
\hline Saúde mental & $-4 \pm 30,56$ & $-12,66 \pm 25,22$ & $9,77 \pm 30,20$ & 0,44 \\
\hline
\end{tabular}

${ }^{*}$ Delta estatisticamente diferente do grupo SE $(p<0,05)$ no teste de Kruskal Wallis. 
calculados. Observou-se que o G40M não diferiu do SE em nenhuma das variáveis. As diferenças entre os deltas indicados devemse, principalmente, às simultâneas reduções da QV no GA35 e aos aumentos do SE. Portanto, outros aspectos independentes ao programa de caminhada possivelmente interferiram nos resultados desses grupos.

Desta forma, considerando apenas o efeito direto do exercício (pré versus pós do G40M), somente os resultados da análise de TE sugerem efeito direto do programa de caminhada sobre um dos domínios da QV: aspectos emocionais.

\section{DISCUSSÃO}

Os resultados dessa pesquisa evidenciam a importância dos estudos de intervenção (experimentais e quaseexperimentais) para a área de exercícios físicos. Isso porque, embora as análises envolvendo somente os grupos experimentais (G40M e GA35) indicassem melhora no G40M versus piora expressiva do GA35, ao observar o grupo SE, foi notável que, na realidade, ocorreu redução em magnitude acentuada dos escores da QV no GA35. Assim, a inclusão do grupo SE viabilizou uma perspectiva mais realista acerca dos efeitos de programas de exercícios sobre a QV.

Além da inclusão do grupo SE, o desenho do presente estudo, que propôs a intervenção com caminhada e não somente comparações entre sujeitos fisicamente ativos e sedentários, por exemplo, também merece destaque. No momento pós-exercício, o grupo de maior assiduidade ao programa de caminhada apresentou escore estatisticamente maior de QV no domínio capacidade funcional. Caso o estudo tivesse desenho observacional e considerasse exclusivamente as análises intergrupos no momento pós, uma conclusão possível seria que sujeitos fisicamente ativos apresentam maior QV no domínio especificado, corroborando com parte importante da literatura prévia composta por estudos observacionais. A abordagem observacional inviabiliza determinar relações de causa e efeito, ou seja, neste tipo de pesquisa não é possível determinar se a QV dos ativos foi maior porque se exercitam ou o contrário, se tem maior motivação para se exercitar porque tem maior QV. Em outras palavras, comparar a QV de sujeitos inativos e ativos é diferente de propor e observar os efeitos de uma intervenção com exercícios. Desta forma, a presente pesquisa se destaca e se diferencia pelo delineamento quaseexperimental.

Ainda em relação aos pontos fortes da pesquisa, outro aspecto que merece destaque foi a população investigada. Apesar de o número de publicações que exploram a relação entre atividade física e QV - seja de forma observacional ou experimental ser expressivo, poucas incluíram brasileiros pertencentes à comunidade universitária, ao grupo etário classificado como adultos jovens ou mesmo a adultos com menos de 50 anos. A maior parte das investigações está centrada no público idoso ou com patologias. Assim, ao reconhecermos a influência da idade e da presença de doenças sobre a QV ${ }^{27-29}$, discutir os resultados da presente pesquisa a partir de estudos conduzidos com pessoas idosas ou que apresentam morbidades não é adequado, pois poderia gerar impressões equivocadas a 
respeito dos resultados apresentados. Neste sentido, foram referenciadas na presente discussão exclusivamente publicações que conduziram pesquisas com voluntários adultos sem patologias. Destaca-se deste contexto que o presente artigo apresenta informações sobre uma população pouco observada em pesquisas de intervenção voltadas à QV.

Apresentados os pontos fortes deste estudo, fica evidente a importância de investigações voltadas a descrever o impacto direto de programas de exercícios na QV da comunidade universitária. Nesta pesquisa o programa de caminhada de oito semanas não teve impacto direto, em comparação ao momento pré-exercício, nos domínios da QV medidos pelo SF-36. Este resultado difere do esperado, visto que parte importante dos estudos prévios sugerem efeito positivo da prática de exercícios sobre a QV na comunidade universitária ${ }^{21,22,25,30}$. Destaca-se, porém, que a maioria destas publicações se caracterizam como observacionais, ou seja, não realizaram intervenção com exercícios; apenas compararam os escores de QV entre grupos de sujeitos organizados segundo nível de atividade física.

Quando considerados exclusivamente os estudos que propuseram intervenções com exercícios este não foi o primeiro a detectar ausência de impacto estatístico sobre a QV entre adultos jovens. Lopes et al. ${ }^{31}$ investigaram o efeito de 12 semanas de Pilates (duas sessões semanais) sobre a QV de 13 mulheres saudáveis, com idade entre 18 e 35 anos. Utilizando o SF-36 para medir a QV, assim como a presente publicação, nenhuma diferença estatística foi encontrada entre os momentos pré e pós intervenção. Uma das principais hipóteses apresentadas pelos autores foi a condição inicial das voluntárias, que demonstraram escores elevados de todos os domínios da $\mathrm{QV}$, muitos dos quais superiores aos valores de referência para a população brasileira para a faixa etária de 25 a 34 anos $^{27}$. Considerando que o SF36 tem o valor de 100 pontos como limite superior, sujeitos que apresentam pontuações elevadas tem pequena margem de melhora. Assim como esses autores, acreditamos que a ausência de mudanças expressivas nos domínios de QV na atual pesquisa também possa ser em parte atribuída aos escores iniciais do grupo G40M.

Ropke $^{29}$ é outro autor que fortalece a discussão em curso no que tange à influência dos escores iniciais de QV sobre o resultado de intervenções com exercícios. Um dos objetivos da pesquisa era compreender o efeito de um programa de Pilates sobre a QV de sujeitos com síndrome da apnéia obstrutiva do sono (SAOS). Para nossa discussão são os dados do grupo controle que merecem atenção, que foi composto por 41 sujeitos saudáveis, com idade média de 51,56 \pm 11,65 anos, submetidos a um programa de exercícios de flexibilidade (2 vezes semanais de 60 minutos), tendo sido observada uma variação positiva para os seguintes domínios avaliados pelo SF-36: vitalidade, aspectos sociais e aspectos emocionais. Dois importantes aspectos de diferença entre a citada pesquisa e a presente publicação são os escores obtidos no momento pré e a idade dos sujeitos. Na presente pesquisa o escore mais baixo do G40M $(51,11 \pm 16,15$ - domínio vitalidade) foi quase 10 pontos mais alto que naquela pesquisa (menor escore: $42,3 \pm 36,5$ 
- domínio limitação por aspectos emocionais). Ao comparar as duas pesquisas quanto ao escore mais alto - domínio limitações por aspectos físicos em ambas - a distância entre as médias é ainda mais significativa (pesquisa atual: 86,11 \pm 18,16; Ropke: 59,1 $\pm 25,4)$. Neste sentido a ausência de efeitos estatísticos da caminhada no G40M pode estar associada aos escores apresentados já no momento inicial (pré-intervenção).

Nesta linha de pensamento, é importante comparar os escores encontrados no momento pré da presente pesquisa, sobretudo para o G40M, com os valores de referência da população brasileira ${ }^{27}$. Dentre os oito domínios avaliados pelo SF-36, quatro estavam próximos à referência nacional considerando as médias das três faixas etárias de até 44 anos descritas no citado artigo: capacidade funcional, aspectos físicos, dor e saúde mental. Todos os demais domínios (estado geral de saúde, vitalidade, aspectos sociais e aspectos emocionais) estavam mais de 10 pontos abaixo do valor referencial. Interessantemente, as médias de todos esses domínios (que estavam inferiores ao valor de referência nacional) se elevaram no G40M, sugerindo que, de fato, escores mais baixos no momento inicial tem maior margem para aumento como resultado de intervenções com exercícios.

Ainda sobre a comparação de escores da QV medidos pelo SF-36 em relação à referência nacional, é válido ressaltar que na comunidade universitária é provável que alguns domínios sejam mais baixos que na população em geral, especialmente, aqueles potencialmente impactados pelos níveis de ansiedade e estresse. Esta questão merece ser investigada em pesquisas futuras, de abrangência nacional. Na mesma linha de raciocínio, ao longo do semestre/ano letivo/acadêmico é provável que ocorram variações na $Q V$, decorrentes das exigências acadêmicas diferenciadas (período de provas, finalização de estágios curriculares e entrega de relatórios finais de pesquisa ao término do semestre letivo, por exemplo), justificando a importância de explorar não somente o impacto de programas de exercício sobre essa variável, como também do próprio ano acadêmico.

As análises dos deltas na presente pesquisa demonstram redução estatisticamente significante em dois domínios da QV para o GA35. Frente à baixa assiduidade dos sujeitos deste grupo ao programa de caminhada, uma hipótese provável, porém, de inviável comprovação, foi que esses tenham vivenciado situações pessoais e/ ou acadêmico-profissionais durante esse período, desencadeando uma redução da $\mathrm{QV}$, o que pode ter contribuído também para a baixa aderência às sessões de exercício.

Em conjunto, as questões até o momento discutidas sugerem que os escores iniciais de QV dos sujeitos, bem como especificidades da vida acadêmica/profissional da comunidade universitária devem ser considerados para a interpretação dos resultados no presente estudo. Em se tratando de um estudo de intervenção, é fundamental que os fatores inerentes ao programa de exercício também sejam discutidos. Sobre a intensidade da caminhada, consideramos possível que aquela aplicada na presente 
pesquisa tenha sido insuficiente para melhorar efetivamente a QV. Durante as 8 semanas de caminhada a intensidade foi elevada de forma progressiva. Somente ao final desse período a intensidade vigorosa foi aplicada. Os resultados encontrados por Mendes Netto $^{30}$ a partir de estudo observacional viabilizam tal discussão sobre o potencial impacto da intensidade empregada. Na publicação citada participaram 352 estudantes de uma Universidade pública do Nordeste do Brasil. Correlações entre a QV (medida pelo WHOQOL-bref) e os níveis de atividade física (medida pelo IPAQ) em diferentes intensidades foram mensuradas utilizando testes estatísticos de Pearson ou Spearman. Correlações significativas $(p<0,05)$ foram encontradas entre a intensidade vigorosa e os domínios físico e psicológico da QV. Já para as intensidades leve e moderada não foram encontradas correlações estatisticamente significantes. Apesar das diferenças metodológicas entre as pesquisas, os resultados desta publicação, em conjunto com aqueles encontrados por Mendes Netto, permitem considerar que intensidades mais altas talvez sejam necessárias para impactar diretamente a QV na comunidade universitária.

Outro aspecto que merece ser discutido frente à literatura prévia é o tipo de exercício empregado, a caminhada. Recentemente Yorks $^{6}$, demonstraram efeito positivo de 12 semanas de aulas em grupos sobre a QV de estudantes universitários. Na pesquisa em questão os voluntários foram organizados em três grupos: exercício em grupos ( $G R ; n=25$ ); exercício sozinho ou com até dois parceiros (SP; tipo musculação ou corrida, por exemplo) $(n=29)$ e controle $(C N ; n=15)$. O primeiro teve um gango estatisticamente significativo nos três domínios da QV (físico, mental e emocional), avaliados por escala visual. No SP somente o domínio mental melhorou. Diante deste resultado, os autores especulam que o componente social do exercício em grupo seja um diferencial para a $\mathrm{QV}$, argumento que também pode fazer parte das explicações dos resultados desta pesquisa.

Sobre a questão das interações sociais proporcionadas pelo exercício, essa pesquisa foi inicialmente planejada para possibilitar sua ampliação. Para tanto, os momentos de aquecimento e de volta a calma foram sempre realizados em grupo, utilizando atividades que geraram interação entre os participantes. Entretanto, devido a baixa assiduidade dos sujeitos, em parte importante das sessões, a interação entre os voluntários foi baixa. Além do contato social proporcionado pelas aulas em grupo, o estudo de York $^{6}$, também se diferencia deste pelo tempo de intervenção: 12 semanas. Assim, é possível que tempo superior a 8 semanas seja necessário para promover benefícios significativos sobre a QV na comunidade universitária, hipótese que também encontra respaldo no estudo observacional de Macedo ${ }^{21}$, que comparou universitários não exercitados com praticantes de exercícios há no mínimo três meses.

Dentre as variáveis inerentes ao exercício que podem ter influenciado os resultados na presente pesquisa, a baixa assiduidade dos voluntários merece destaque. A média de assiduidade no grupo G40M foi de $59,44 \pm 14,46 \%$, variando de 40 a $85 \%$. Assim, semanalmente este grupo realizou a caminhada, em média 1,6 vezes por semana, 
frequência que pode ter sido insuficiente para benefícios mais expressivos sobre a QV. Esta hipótese encontra respaldo na pesquisa de Silva et al. ${ }^{22}$ que analisaram as relações entre o nível de atividade física (NAF) e a QV (medida pelo WHOQOL-bref) de professores, servidores e estudantes da Universidade Católica de Pelotas. Os 863 indivíduos participantes do estudo foram organizados nos grupos moderadamente ativo $(n=313)$, inativo $(n=210)$, ativo $(n=207)$ e muito ativo $(n=136)$. O último apresentou escores significativamente maiores para a QV em relação ao grupo de inativos nos domínios: físico, psicológico e meio ambiente. Neste contexto, entende-se que a QV da comunidade universitária tende a ser maior quanto maior o tempo semanal empregado em atividades físicas. Portanto, a assiduidade dos voluntários no programa de caminhada foi provavelmente um importante limitante para aumentos expressivos da QV na presente pesquisa.

Diante deste cenário, destaca-se a necessidade de compreender melhor a questão da aderência em programas de exercícios físicos na comunidade universitária, pois, grande parte dos estudantes vivencia durante os anos de formação universitária um período de aquisição ou ampliação de hábitos não-saudáveis ${ }^{11}$. Ademais, o aumento das responsabilidades e da carga horária de estudos e/ou trabalho, frequentemente, tornam o cotidiano estressante ${ }^{10}$. Nesse sentido, programas que incentivam a prática de atividades físicas durante os anos de vida universitária têm potencial impacto sobre a saúde desses sujeitos. É relevante que em pesquisas futuras envolvendo programas de exercício sejam incluídos dados que permitam acessar barreiras à aderência, especialmente entre os desistentes.

Outras variáveis que precisam ser exploradas futuramente, visto que a presente pesquisa viabiliza apenas especular sobre elas, são a modalidade, intensidade, frequência semanal e o tempo total de programas de exercício sobre a QV. Assim, identificamos como limitações principais do presente estudo: a baixa assiduidade dos sujeitos, o curto tempo de intervenção (8 semanas), a não separação dos sujeitos em grupos de estudantes e servidores e a ausência de uma análise qualitativa que viabilizasse compreender de forma mais clara os aspectos que geraram redução numérica em alguns domínios da QV em sujeitos do grupo GA35.

\section{CONCLUSÃO}

Oito semanas de caminhada foram insuficientes para promover melhora estatística dos domínios da QV, avaliados pelo questionário SF-36. No grupo composto por sujeitos que tiveram assiduidade igual ou superior a $40 \%$ no programa de caminhada (G40M) o domínio aspectos emocionais foi o que sofreu mudança numérica mais expressiva, indicada pelo tamanho do efeito, embora sem diferença estatística nas análises pareadas. Para o domínio capacidade funcional, o G40M apresentou, em comparação aos demais grupos, escore estatisticamente superior no momento pós, viabilizando especular que períodos mais longos de intervenção são necessários para gerar efeito direto dos exercícios em tal variável. 
No grupo com baixa assiduidade ao programa de caminhada (GA35), dentre os oito domínios da QV, seis sofreram redução nas médias entre momentos pré e pós, sem diferença estatística. Quando tais resultados são comparados àqueles observados nos demais grupos, emerge a hipótese que fatores não mensurados (pessoais, acadêmicos e/ ou profissionais), possivelmente afetaram a QV nesse grupo ao longo do período de intervenção, os quais podem ter afetado também a assiduidade aos exercícios. Nesse contexto, visando favorecer compreensão mais aprofundada sobre essa temática na população universitária, é interessante que pesquisas futuras incluam estratégias e metodologias que considerem perspectivas qualitativas de análise.

Apesar do impacto não expressivo da intervenção realizada sobre a $Q V$, a presente pesquisa tem como importante

\section{REFERÊNCIAS}

1. Ruegsegger GN, Booth FW. Health Benefits of Exercise. Cold Spring Harb Perspect Med. 2018; 8(7):a029694.

2. Grazioli E, Dimauro I, Mercatelli N, Wang G, Pitsiladis Y, Di Luigi L, Caporossi D. Physical activity in the prevention of human diseases: role of epigenetic modifications. BMC Genomics. 2017;18(Suppl 8):802.

3. Warburton DER, Bredin SSD. Health benefits of physical activity: a systematic review of current systematic reviews. Curr Opin Cardiol. 2017; 32(5):541-556.

4. Panza GA, Taylor BA, Thompson PD, White CM, Pescatello LS. Physical activity intensity and subjective well-being in healthy adults. J Health Psychol. 2019; 24(9):1257-1267. contribuição à comunidade científica a demonstração prática a respeito da relevância de estudos de desenho experimental para explorar e compreender melhor as relações entre a prática de atividade física e a QV, sobretudo na comunidade universitária. A existência de um grupo não exercitado (SE), composto por membros dessa comunidade, se mostrou fundamental para evitar que fosse superestimado o efeito do programa de caminhada sobre a QV.

Destaca-se ainda que fatores relacionados ao programa de exercício proposto (modalidade, intensidade, volume, tempo total de intervenção e assiduidade dos participantes) e outros independentes desse (escores iniciais de QV, momentos de realização das coletas em relação ao semestre acadêmico) podem ter exercido influência nos resultados e merecem ser acessados em estudos futuros.
5. Nowak PF, Bozek A, Blukacz M. Physical Activity, Sedentary Behavior, and Quality of Life among University Students. BioMed R Inter. 2019; 2019(ID9791281):1-10.

6. Yorks DM, Frothingham CA, Schuenke MD. Effects of Group Fitness Classes on Stress and Quality of Life of Medical Students. J Am Osteopath Assoc. 2017; 117(11):17-25.

7. World Health Organization. WHO Guidelines on physical activity and sedentary behaviour. 2020 Disponível em: https://www.who.int/publications/i/ item/9789240015128. Acesso em: 16/02/2021.

8. Couto DAC, Martin DRS, Molina GE, Fontana KE, Junqueira Jr LF, Porto LGG, Nível insuficiente de atividade física se associa a menor qualidade de vida e 
ao estudo noturno em universitários do Distrito Federal. Rev Bras Cien Esp. 2019;41(3):322-330.

9. Castillo-Retamal F, Castillo-Retamal M, Vásquez-Gómez J, Cordero-Tapia F, Both J, Oliveira APB. Gasto energético e nível de atividade física em acadêmicos e funcionários universitários. Revi Horizonte Ciências de la Actividad Física. 2019;10(1):1-10.

10. Oliveira ES, Silva AFR, Silva KCB, Moura TVC, Araújo $A L$, Silva $A R V$. Estresse e comportamentos de risco à saúde entre estudantes universitários. Rev Bras Enferm. 2020;73(1): e20180035.

11. Sánchez-Ojeda MA, De Luna-Bertos E. Hábitos de vida saludable en la población universitaria. Nutr Hosp. 2015; 31(5):1910-1919.

12. Soto Ruiz MN, Aguinaga Ontoso I, Guillén-Grima F, Marín Fernández $\mathrm{B}$. Changes in the physical activity of university students during the first three years of university. Nutr Hosp. 2019;36(5):1157-1162.

13. Dos Santos LR, Brito ECC, Lira Neto JCG, Alves LEP, Alves LRA, Freitas RWJF. Análise do sedentarismo em estudantes universitários. Rev Enferm UERJ. 2014 22(3):416-21.

14. Ascefl BO, Haddad JPA, Álvares J, Junior AAG, Costa $\mathrm{EA}$, Acurcio FA, et al. Qualidade de vida relacionada à saúde dos usuários da atenção primária no Brasil. Rev Saúde Publica. 2017; 51(Supl):2-22s.

15. Boggatz T. Quality of life in old age: a concept analysis. Int J Older People Nurs. 2016;11(1):55-69.

16. Karimi M, Brazier J. Health, Health-Related Quality of Life, and Quality of Life: What is the Difference? Pharmacoeconomics. 2016; 34(7):645-9.

17. Haraldstad K, Wahl A, Andenæs R, Andersen JR, Andersen $\mathrm{MH}$, Beisland $\mathrm{E}$, et al. A systematic review of quality of life research in medicine and health sciences. Qual Life Res. 2019; 28:2641-50.

18. WHOQOL. The World Health Organization Quality of Life assessment (WHOQOL): position paper from the World Health Organization. Soc Sci Med. 1995; 41(10):1403-9.

19. Minayo MC. Qualidade de vida e saúde: um debate necessário. Rio de Janeiro: Ciência \& Saúde Coletiva, 2000.

20. Scattolin FAA. Qualidade de vida: a evolução do conceito e os instrumentos de medida. Rev da Facul de Ciên Méd de Sorocaba, 2007; 8(4):1-5.

21. Macedo CSG, Garavello JJ, Oku EC, Garavello FH, Agnoll PD, Nocetti PM. Benefícios do exercício físico para a qualidade de vida. Rev Bras de Ativ Fís \& Saúde. 2003; 8(2):19-27.

22. Silva RS, Silva I, Silva RA, Souza L, Tomasi E. Atividade física e qualidade de vida. Ciênc saúde coletiva. 2010;15(1):115-20

23. Medeiros AGA, Tomaz RS, Carvalho KS, Santos SR, Almeida ILS. Contribuições da ginástica laboral para qualidade de vida dos servidores de uma universidade estadual. Extensio: Rev Eletr de Extensão. 2019;16(32):107-115.

24. American College of Sports Medicine. Diretrizes do ACSM para os testes de esforço e sua prescrição. $6^{\mathrm{a}}$.ed. Rio de Janeiro: Guanabara Koogan, 2003.

25. Ciconelli RM, Ferraz MB, Santos W, Meinão I, Quaresma MR. Tradução para a língua portuguesa e validação do questionário genérico de avaliação de qualidade de vida SF-36 (Brasil SF-36). Rev Bras Reumatol. 1999; 39(3):143-50.

26. Espírito-Santo H, Daniel F. Calcular e apresentar tamanhos do efeito em trabalhos científicos (1): As limitações do $p<0,05$ na análise de diferenças de médias de dois grupos. Rev Portu de Invest Comport e Social. 2015;1(1): 3-16

27. Laguardia J, Campos MR, Travassos C, Najar AL, Anjos LA, Vasconcellos MM. Dados normativos brasileiros do questionário Short Form-36 versão 2. Rev Bras Epidemiol. 2013; 16(4):889-897.

28. Noronha DD, Martins AMEBL, Dias DS, Silveira MF, Paula AMB, Haikal DS. Qualidade de vida relacionada à saúde entre adultos e fatores associados: um estudo de base populacional. Ciênc saúde coletiva. 2016; 21(2):463-474.

29. Ropke LM, Avaliação do efeito de um programa de exercícios do método Pilates na qualidade do sono e na qualidade de vida de pacientes com SAOS, em uso adequado de CPAP, [Dissertação de Mestrado]. Botucatu: Faculdade de Medicina, Universidade Estadual Paulista Júlio de Mesquita Filho; 2017. (84p).

30. Mendes-Netto RS, Silva CS, Costa D, Raposo OFF. Nível de atividade física e qualidade de vida de estudantes universitários da área de saúde. Rev Bras de Ciên da Saúde. 2012;10(34):47-55. 
31. Lopes TP, Kuster PPS, Sarro KJ, Campos JL, Silva WRT, Vancini RL.. Efeitos do Pilates solo na qualidade de vida de mulheres saudáveis ativas. Conexões: Educ Fís, Esp e Saúde, 2019;17,e019026:1-13.

\section{CORRESPONDÊNCIA}

Tatiane Gomes Teixeira

Avenida Amazonas, 1239

Condomínio Leonardo da Vinci Spazio Club,

apartamento 1301, bairro Nova Porto Velho, Porto Velho,

Rondônia, Brasil. CEP: 76804-171.

E-mail: tatiane.teixeira@unir.br 